\title{
MiR-339 is a potential biomarker of coronary heart disease to aggravate oxidative stress through Nrf2/FOXO3 targeting Sirt2
}

\author{
Linying Shi, Yuan Zhang, Jing Zhang, Yuanfeng Gao, Jiamei Liu, Mulei Chen, Xinchun Yang \\ Department of Cardiology, Beijing Chaoyang Hospital, Capital Medical University, Beijing, China \\ Contributions: (I) Conception and design: L Shi, X Yang, M Chen; (II) Administrative support: Y Zhang, J Zhang; (III) Provision of study materials or \\ patients: Y Gao, J Liu; (IV) Collection and assembly of data: L Shi, Y Zhang; (V) Data analysis and interpretation: L Shi, Y Zhang; (VI) Manuscript \\ writing: All authors; (VII) Final approval of manuscript: All authors. \\ Correspondence to: Mulei Chen; Xinchun Yang. Department of Cardiology, Beijing Chaoyang Hospital, Capital Medical University, Gongtinanlu Road \\ No.8, Chaoyang District, Beijing 100020, China. Email: cml68@sina.cn; Xinchunyang802419@163.com.
}

\begin{abstract}
Background: MicroRNA (miRNA) is a class of small-molecule RNA that can regulate gene expression at post-transcription level. It is involved in the genesis and development of multiple diseases. The aim of this paper was to explore the mechanisms of miR-339 in coronary heart disease (CHD).

Methods: In this study, we enrolled patients with CHD from Beijing Chaoyang Hospital and performed animal experiments on CHD rats. In vitro experiments, such as histopathologic assay, quantitative real-time PCR assay, luciferase reporter assay, western blotting assay, and immunofluorescence assay were carried out to characterize the contents and associations of miR-339, Nrf2, FOXO3, and Sirt2 in CHD samples and cells. In vivo model was also established on rats.
\end{abstract}

Results: In CHD rat, miR-339 was up-regulated compared with control group. The expression of miR339 up-regulation increased oxidative stress in vitro model via suppression of Sirt2/Nrf2/FOXO3. However, down-regulation of miR-339 expression inhibited oxidative stress in vitro model via activation of Sirt2/Nrf2/ FOXO3. The Sirt2 or Nrf2 inhibitor reduced the protective effect of miR-339 down-regulation on oxidative stress in vitro model.

Conclusions: Down-regulation miR-339 may be the new targets to treat CHD through Nrf2/FOXO3 targeting Sirt2, and miR-339 may be a potential biomarker of CHD.

Keywords: miR-339; Nrf2; FOXO3; Sirt2; coronary heart disease (CHD)

Submitted Mar 10, 2020. Accepted for publication Sep 25, 2020.

doi: $10.21037 /$ apm-20-603

View this article at: http://dx.doi.org/10.21037/apm-20-603

\section{Introduction}

Coronary heart disease (CHD) is a cardiovascular system disease with high morbidity. It is an organic lesion resulting from luminal occlusion and stenosis due to coronary atherosclerosis and thereby induces myocardial ischemia (1). CHD has severely threatened the health and life quality of the middle-aged and elderly patients (2). The stenosis of coronary atherosclerosis can reflect the extent of CHD (2). The pathogenic factors of CHD include diabetes, hypertension, obesity and unhealthy lifestyle (smoking, drinking, uptake of high-calorie food, lack of exercise, and psychological factors) (3). CHD has been extensively studied in clinic recently (4). It is discovered that it is susceptible to multiple factors, including family history, unhealthy lifestyle and smoking. Research indicates that oxidative stress, inflammatory response, and vascular endothelial dysfunction play vital roles in the genesis and development of CHD $(5,6)$.

Many studies suggested that CHD is closely related to the Sirtuin family during the genesis and development of its pathogenesis (7). Moreover, the Sirtuin family is also closely correlated with the risk factors of cardiovascular diseases, such as glucolipid metabolism. Sirtuin family is 
a class of silence information regulators, which include 7 family members, from SIRT1-7 (7). However, recent studies find that SIRT2 has similar or contrary function in cardiovascular disease and its pathogenesis (7).

Recent findings have indicated that miRNA is related to the genesis and development of atherosclerosis $(8,9)$. miRNA is extensively involved in the whole process of atherosclerosis (10). They include LDL deposition under the endarterium that leads to endothelial disturbance, cytokine release, monocyte adhesion and migration, monocyte transformation into macrophage, protective phagocytosis of macrophage, foam cell formation, inflammation amplification of macrophage and foam cell, smooth muscle cell proliferation and migration, as well as synthesis and degradation of fibrous cap matrix (8). Thus, miRNA plays a key role in the pathophysiological processes such as vascular endothelial dysfunction, inflammation, cell apoptosis, angiogenesis, atherosclerosis, neointima hyperplasia or restenosis (11). The aim of this paper was to explore the mechanisms of miR-339 in CHD. We present the following article in accordance with the ARRIVE reporting checklist (available at http://dx.doi.org/10.21037/ apm-20-603).

\section{Methods}

\section{Patient with CHD}

Patients with CHD and normal healthy volunteers were collected from Beijing Chaoyang Hospital, Capital Medical University and electrocardiogram of patient with CHD proceeded. The patients were diagnosed as CHD with angiographic evidence of at least one segment of a major coronary artery, including the left anterior descending, left circumflex, or right coronary artery, with $>50 \%$ organic stenosis. The patients were included in this study if they have no family history of CHD and no history of significant concomitant diseases, including hepatic failure, renal failure, hepatitis, cardiomyopathy, congenital heart disease, bleeding disorders, previous thoracic irradiation therapy, and malignant diseases. Serum samples of patient with CHD and normal healthy volunteers were collected and saved at $-80{ }^{\circ} \mathrm{C}$. The experiments in the present study were approved by Beijing Chaoyang Hospital, Capital Medical University.

The study was conducted in accordance with the Declaration of Helsinki (as revised in 2013). The study was approved by ethics board of Beijing Chaoyang Hospital,
Capital Medical University (No.: 2016-6-4-1). Informed consent was obtained from every participant.

\section{Animals and ethical approval}

Wistar rats divided into two groups, normal control $(n=6)$ and CHD model $(n=6)$. CHD model rat was achieved by feeding with high-fat diet for 12 weeks and normal control rats were achieved by feeding with normal diet for 12 weeks. Experiments were performed under a project license granted by ethics board of Beijing Chaoyang Hospital, Capital Medical University (approval No.: F2016-025). Animals were treated in accordance with Guide for the Care and Use of Laboratory Animals (8th edition, National Academies Press).

\section{Histopatbologic assay}

The heart tissues were fixed with $4 \%$ paraformaldehyde for $24 \mathrm{~h}$. Tissue samples were embedded in paraffin, and the 5 - $\mu \mathrm{m}$ - thick sections were stained with hematoxylin-eosin (H\&E). Tissue samples were obtained using fluorescence microscopy (Nikon Eclipse TE2000-U, Japan).

\section{Quantitative real-time PCR assay}

The total RNA samples from mice were obtained using TransZol according to the manufacturer's protocol (Beijing TransGen Biotech Co., Ltd. Beijing, China). Each RNA sample was reverse transcribed into cDNA using the kit of TransStart Top Green qPCR SuperMix (Beijing TransGen Biotech Co., Ltd. Beijing, China). Real-time PCR amplification was performed using the STEP ONE Real-time PCR Detection System (Foster City, CA, USA) with the SYBR Green master mix (Applied Biosystems, Foster City, CA, USA). The cycling conditions were $95{ }^{\circ} \mathrm{C}$ for $10 \mathrm{~min}$ followed by 40 cycles of $95{ }^{\circ} \mathrm{C}$ for $15 \mathrm{~s}$ and $60{ }^{\circ} \mathrm{C}$ for $30 \mathrm{~s}$

Measurement of intracellular ROS. H9C2 cells were stained with DCFH-DA $(10.0 \mu \mathrm{M})$ for $30 \mathrm{~min}$ at $37^{\circ} \mathrm{C}$, and ROS levels were analyzed using fluorescence microscopy (Nikon Eclipse TE2000-U, Japan).

\section{Luciferase reporter assay}

Sirt2 plasmids were synthesized into luciferase reporter plasmid (pMIR-REPORT) (Promega Corporation, Fitchburg, WI, USA). Sirt2 plasmids and miR-339 mimics 
were co-transfected into $\mathrm{H} 9 \mathrm{C} 2$ cells using Lipofectamine 2000 (Thermo Fisher Scientific, Waltham, MA, USA) according to the manufacturer's protocol. $48 \mathrm{~h}$ after transfection, luciferase activities were measured using the Dual-Luciferase Assay (Promega Corporation) according to the manufacturer's instruction.

\section{Enzyme-linked immunosorbent assay (ELISA)}

The cell was collected and used to measure MDA, SOD, GSH, GSH-PX and ROS levels using ELIST kits according to the manufacturer's protocol. Absorbance values were measured using a microplate reader (Multiskan MK33; Thermolab systems, Helsinki, Finland) at $450 \mathrm{~nm}$.

\section{Western blotting assay}

The total protein samples from the cells were homogenized using RIPA lysis buffer and the protein concentrations of the samples were determined using a BCA Protein Assay Kit. The proteins were separated by SDS-PAGE (10\%) and then transferred to PVDF membranes (Millipore, Massachusetts, USA). Membranes were blocked with $5 \%$ non-fat in milk for $1 \mathrm{~h}$ at $37^{\circ} \mathrm{C}$ and incubated with Sirt2, Nrf2, HO-1, FOXO3 and GAPDH (Cell Signaling Technology Inc.) at $4{ }^{\circ} \mathrm{C}$ overnight. Membranes were washed with TBST for $15 \mathrm{~min}$ and incubated with the antirabbit or anti-mouse secondary antibody $(1: 5,000$, Cell Signaling Technology Inc.) for $1 \mathrm{~h}$ at $37^{\circ} \mathrm{C}$.

\section{Immunofluorescence assay}

The cell was washed with PBS and fixed with $4 \%$ paraformaldehyde for $15 \mathrm{~min}$. The cell was incubated with $5 \%$ BSA+ $0.2 \%$ TrisX100 in TBST for $1 \mathrm{~h}$ at room temperature and incubated with Sirt2 (1:100, Cell Signaling Technology Inc.) at $4{ }^{\circ} \mathrm{C}$ overnight. The cell was stained with 555 - anti-rabbit secondary antibody for $1 \mathrm{~h}$ at $37{ }^{\circ} \mathrm{C}$ and stained with DAPI assay for $15 \mathrm{~min}$ at darkness. Cell samples were imaged by fluorescence microscopy (Nikon Eclipse TE2000-U, Japan) at 200× magnification.

\section{Vitro model of CHD}

The H9C2 cells were purchased from the Chinese Academy of Medical Sciences, Shanghai, China. H9C2 cells were maintained in DMEM supplemented with $10 \%$ FBS in a humidified atmosphere of $5 \% \mathrm{CO}_{2}$ and $95 \% \mathrm{O}_{2}$ at
$37{ }^{\circ} \mathrm{C}$. MiR-339, anti-miR-339, and negative mimics were transfected into cell using Lipofectamine 2000 (Thermo Fisher Scientific, Waltham, MA, USA) according to the manufacturer's protocol. They were subjected to hypoxia using DMEM/F12 without FBS and glucose in a hypoxia chamber (Forma 370; Thermo Fisher Scientific, Waltham, MA, USA) saturated with a gas mixture $\left(95 \% \mathrm{~N}_{2}\right.$ and $5 \%$ $\mathrm{CO}_{2}$ ) at $37^{\circ} \mathrm{C}$.

\section{Statistical analysis}

Data are presented as the mean \pm SD using SPSS 17.0. Student's $t$-test or one-way analysis of variance (ANOVA) and Tukey's post-test was used to analyze the statistical significance. Statistical significance was considered to be $\mathrm{P}<0.05$.

\section{Results}

\section{The expression of miR-339 in patients or rats with CHD}

Firstly, we analyzed the changes in microRNAs in patients or rats with CHD. As showed in Figure 1A,B,C, electrocardiogram showed sinus arrhythmia, and we found that the serum of miR-339 expression was increased in patients with CHD, compared with normal group. Then, in rats with CHD, SOD, GSH, and GSH-PX levels were inhibited, and MDA level was increased, compared with normal rat group (Figure 1D,E,FG). HE staining showed that a large number of blood clots were formatted in rats with CHD, compared with normal group (Figure 1H). The serum of miR-339 expression was increased in rats with CHD, compared with normal group (Figure 1I). These results showed that miR-339 may be keeping in the formation of CHD.

\section{Over-expression of miR-339 increased oxidative stress and not affected on ROS levels in vitro model}

This study tested the function of miR-339 on the oxidative stress in vitro model. There was increase the expression of miR-339 in miR-339 mimics, compared with negative group (Figure $2 A$ ). Over-expression of miR-339 increased MDA levels, and inhibited SOD, GSH and GSH-PX levels in vitro model, compared with negative group (Figure 2B,C,D,E). However, over-expression of miR-339 not affected ROS level in vitro, compared with negative group (Figure 2F,G). 
A

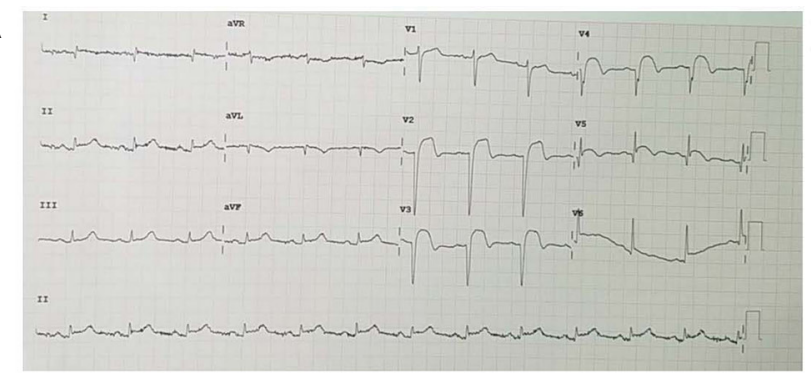

C
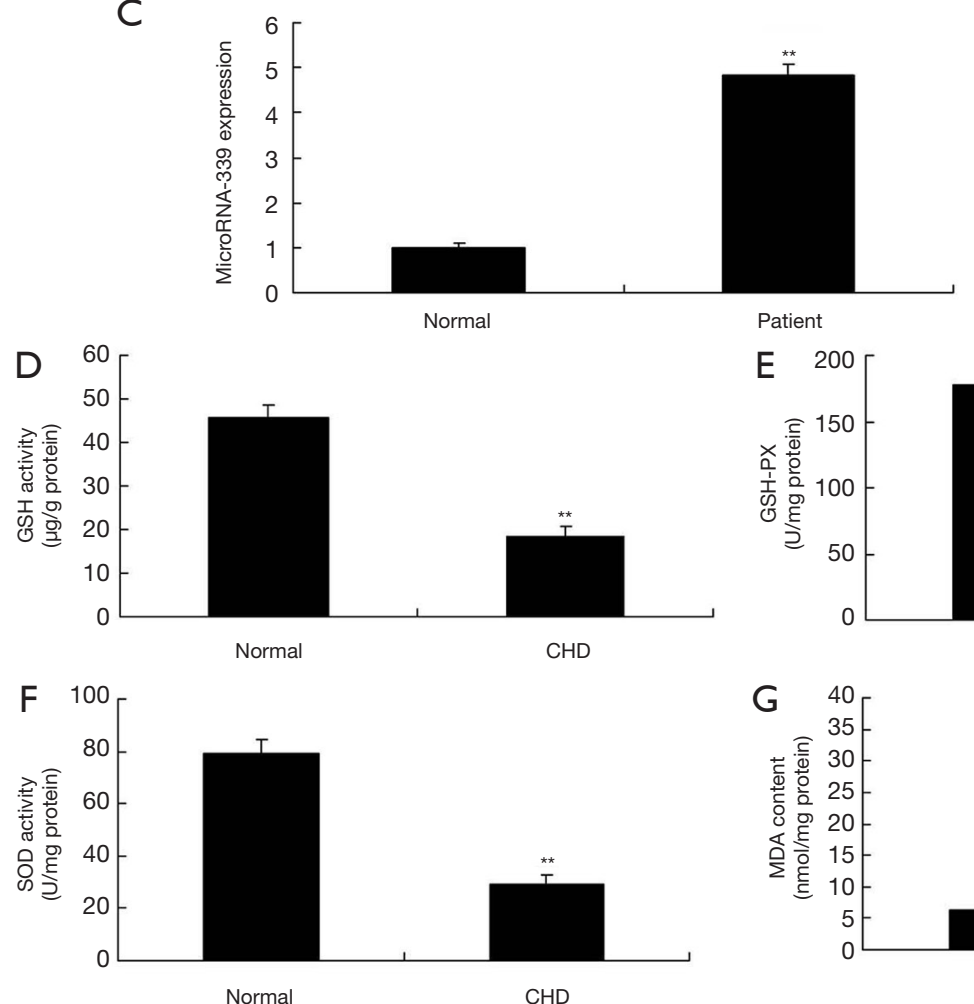

$\mathrm{H}$

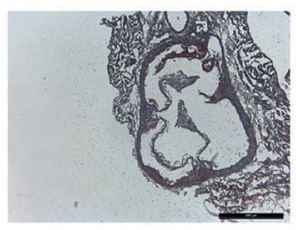

Normal

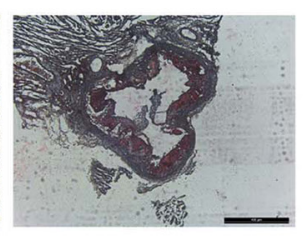

$\mathrm{CHD}$

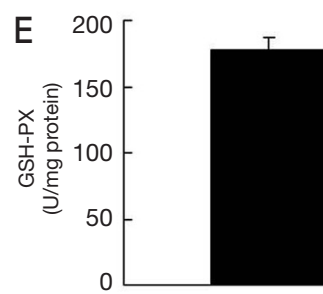

Normal

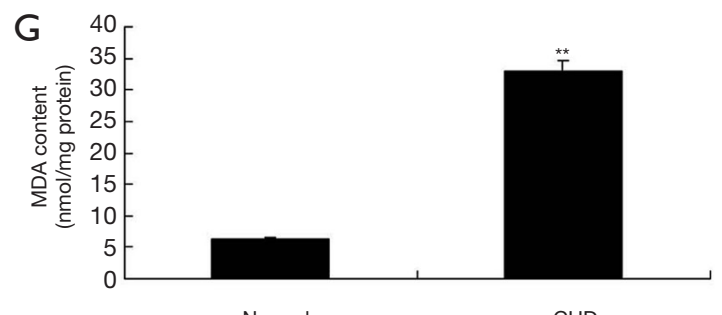

Normal

$\mathrm{CHD}$

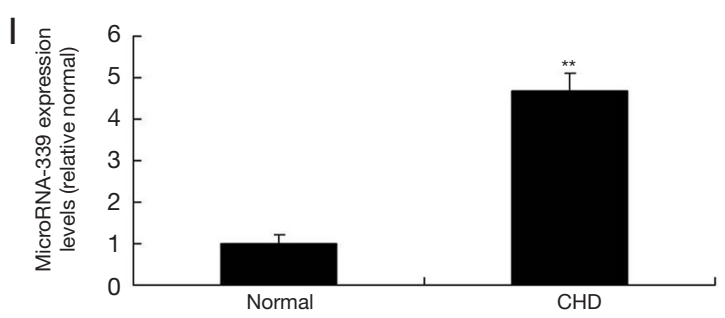

Figure 1 The expression of miR-339 in patients or rat with CHD. Electrocardiogram for patients with CHD (A), gene chip and QPCR for miR-339 expression (B,C) in patients with CHD; GSH (D), GSH-PX (E), SOD (F), MDA (G) level, HE staining (H) and QPCR for miR-339 expression (I) in rat with CHD. Normal, normal volunteer or normal rat group; patient, patients with CHD group; CHD, the rat with CHD group. ${ }^{* *}, \mathrm{P}<0.01$ compared with normal volunteer or normal rat group. CHD, coronary heart disease; GSH, glutathione; SOD, superoxide dismutase; MDA, malondialdehyde. 

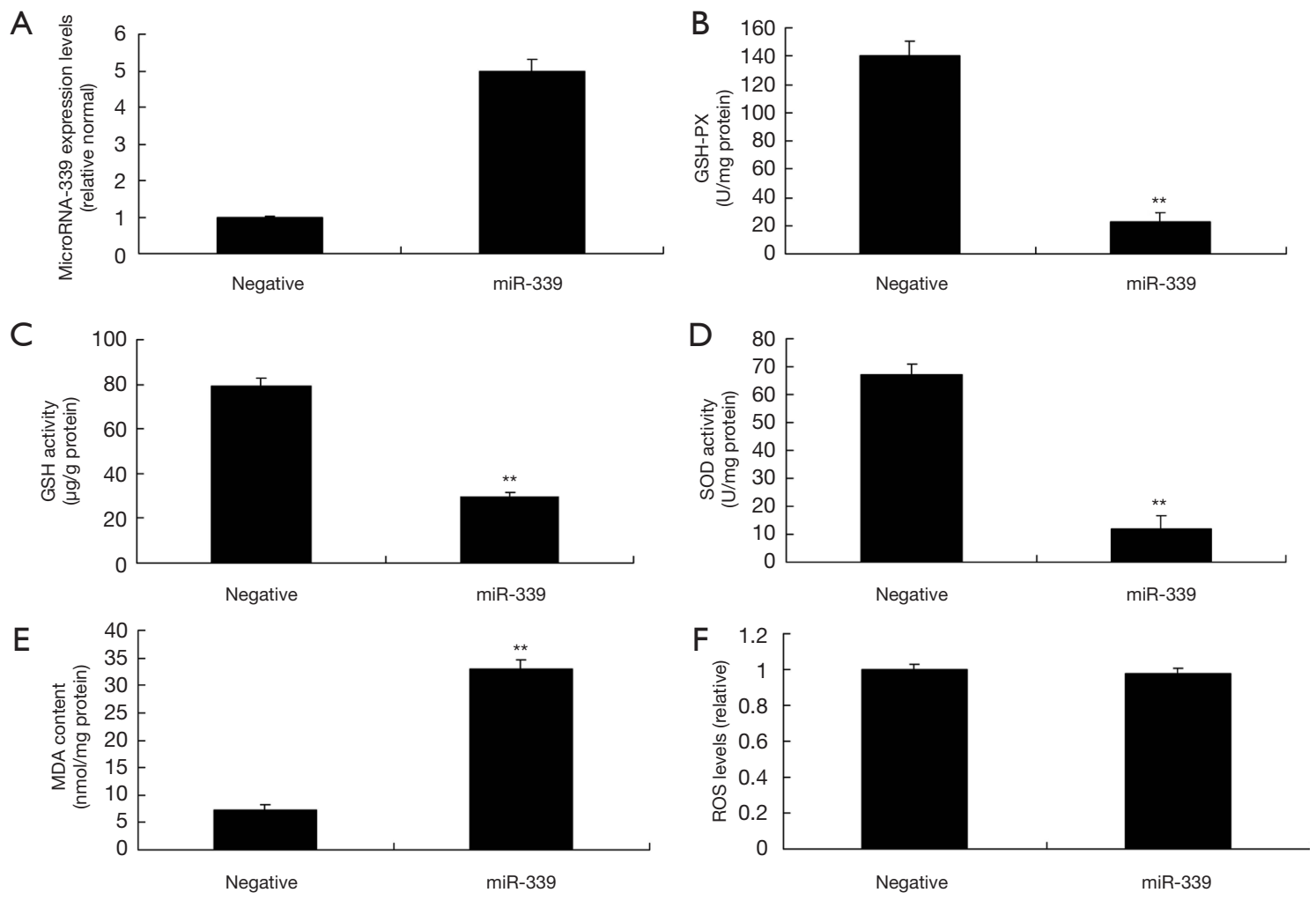

G
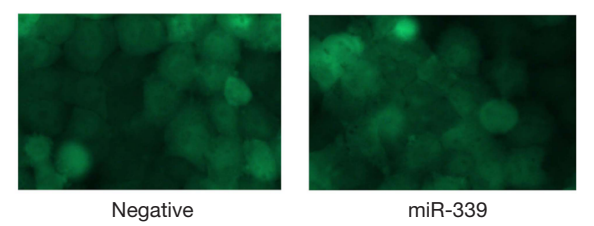

Figure 2 Over-expression of miR-339 increased oxidative stress and not affected on ROS levels in vitro model. QPCR for miR-339 expression (A), GSH (B), GSH-PX (C), SOD (D), MDA (E), ROS (F,G) levels. Negative, negative mimics group; miR-339, overexpression of the miR-339 group. ${ }^{* *} \mathrm{P}<0.01$ compared with negative mimics group. GSH, glutathione; SOD, superoxide dismutase; MDA, malondialdehyde; ROS, reactive oxygen species.

\section{Down-regulation of miR-339 expression inhibited oxidative stress and not affects ROS levels in vitro model}

To validate the effect of miR-339 on oxidative stress in vitro model, we used anti-miR-339 to reduce the expression of miR-339 in vitro model. As showed in Figure $3 A$, there was inhibition of miR-339 expression in vitro model, compared with negative group. Meanwhile, we also found that downregulation of miR-339 reduced MDA level, and increased SOD, GSH and GSH-PX levels in vitro model, compared with negative control group (Figure $3 B, C, D, E$ ). However, down-regulation of miR-339 not regulates ROS levels in vitro model, compared with negative group (Figure 3F,G).
So, these results showed that miR-339 only regulates oxidative stress and not affect ROS levels in vitro model of CHD.

\section{MiR-339 regulates Sirt2/Nrf2/FOXO3 in vitro model of CHD.}

We explored the mechanism of miR-339 on oxidative stress in CHD model. Over-expression of miR-339 suppressed Sirt2, Nrf2, HO-1 and FOXO3 mRNA expressions in vitro model, compared negative group (Figure 4A,B,C,D,E). Luciferase reporter assay showed that miR-339 targets Sirt2 mRNA and luciferase reporter activity levels were reduced 
A

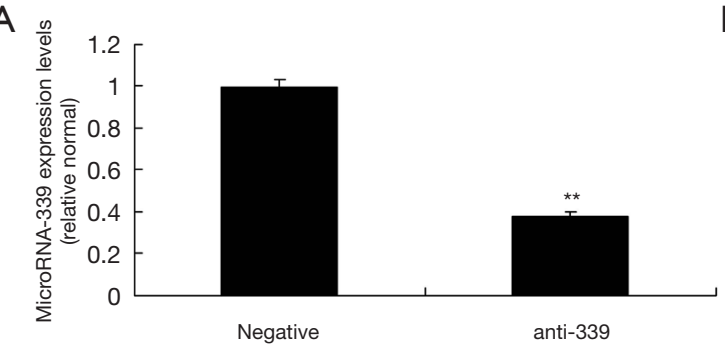

C

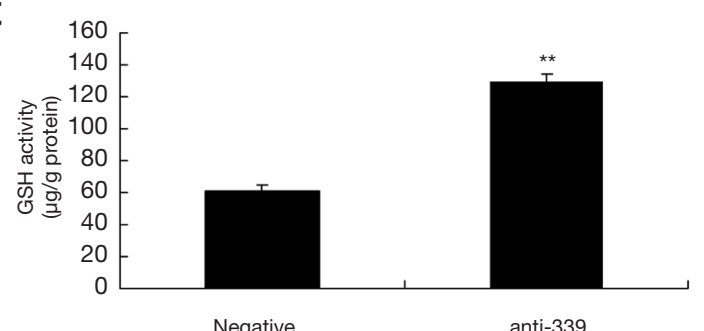

$\mathrm{E}$

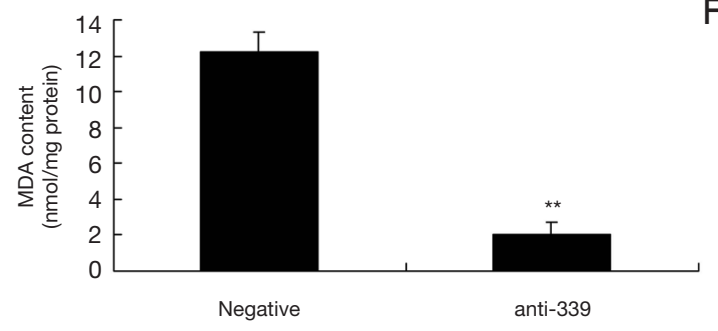

G

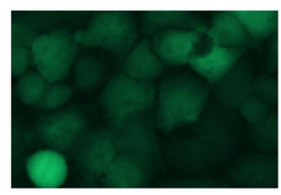

Negative
B

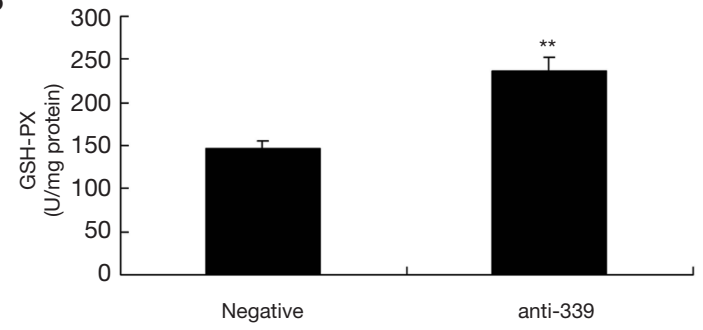

D

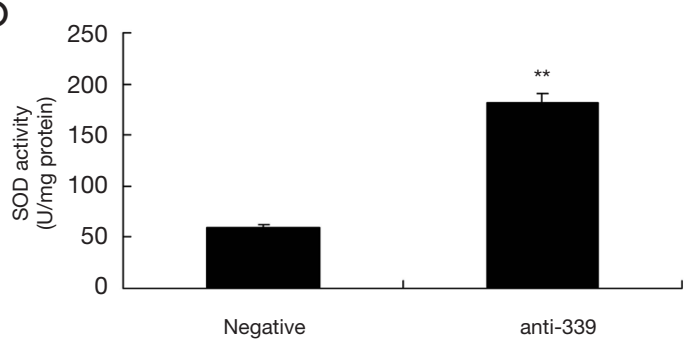

$\mathrm{F}$

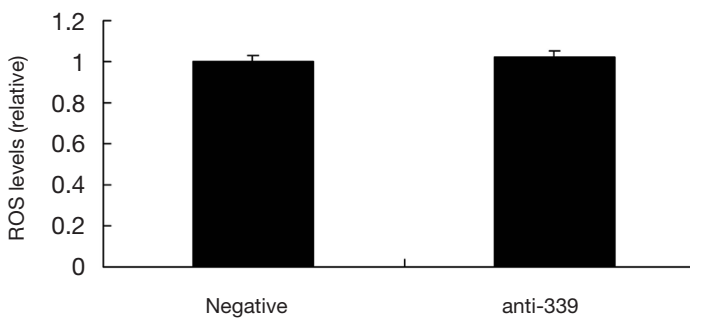

Figure 3 Down-regulation of miR-339 expression inhibited oxidative stress and not affects ROS levels in vitro model. QPCR for miR339 expression (A), GSH (B), GSH-PX (C), SOD (D), MDA (E), ROS (F,G) levels. Negative, negative mimics group; anti-339, downregulation of miR-339 group. **, $\mathrm{P}<0.01$ compared with negative mimics group. GSH, glutathione; SOD, superoxide dismutase; MDA, malondialdehyde; ROS, reactive oxygen species.

in over-expression of miR-339 group, compared with negative group (Figure 4F, G). IF showed that miR-339 overexpression suppressed the protein expression of Sirt2 in vitro model group, compared with negative group (Figure 4H). Then, we also found that over-expression of miR-339 suppressed Sirt2, Nrf2, HO-1 and FOXO3 protein expressions in vitro model, compared negative group (Figure $5 A, B, C, D, E)$. Down-regulation of miR-339 induced Sirt2, $\mathrm{Nrf2}, \mathrm{HO}-1$ and $\mathrm{FOXO} 3$ protein expressions in vitro model, compared negative group (Figure $5 F, G, H, I, 7$ ). These results showed that miR-339 regulates Sirt2/Nrf2/FOXO3 to regulate oxidative stress in vitro.
The Sirt2 inhibitor reduced the protective effect of miR339 down-regulation on the oxidative stress in vitro model

Next, this study analyzed the role of Sirt2 in the function of miR-339 on oxidative stress in CHD model. Sirt2 inhibitor could suppress the protein expression of Sirt2, Nrf2, HO-1 and $\mathrm{FOXO} 3$ protein expressions in vitro model by downregulation of miR-339, compared with miR-339 downregulation group (Figure $6 A, B, C, D, E$ ). The inactivation of Sirt2 reduced the protective effect of miR-339 on the inhibition of MDA levels, and induction of SOD, GSH and GSH-PX levels in vitro model of CHD, compared with down-regulation of miR-339 group (Figure 6F,G,H,I). 
A

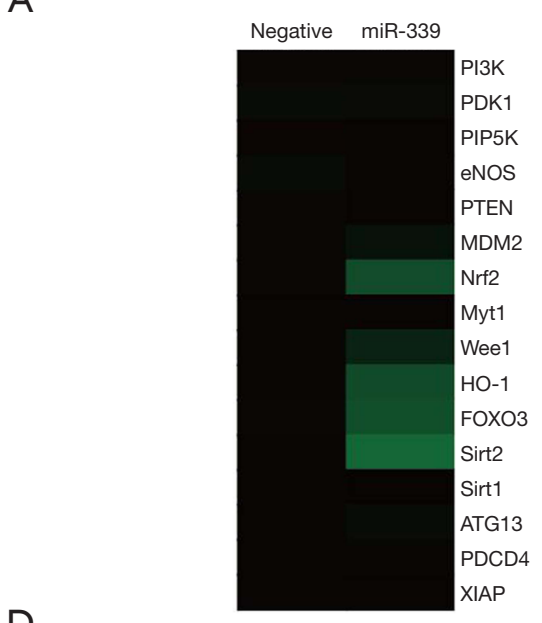

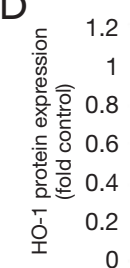

$\mathrm{F}$

MicroRNA-339---GCACUCGAGGACCUCCUGUCCCU Sirt2---UUUUAACUCUUCCAGGACAGGGA

$\mathrm{H}$

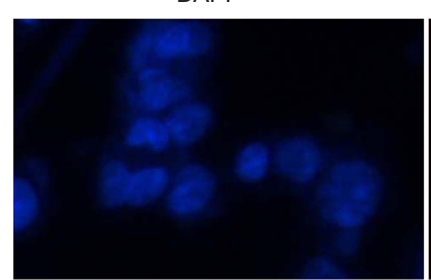

DAPI

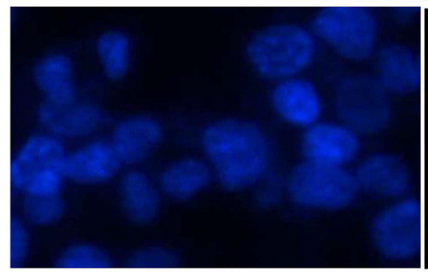

B

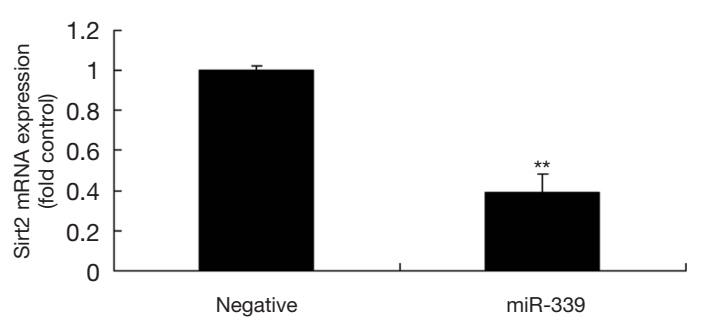

C

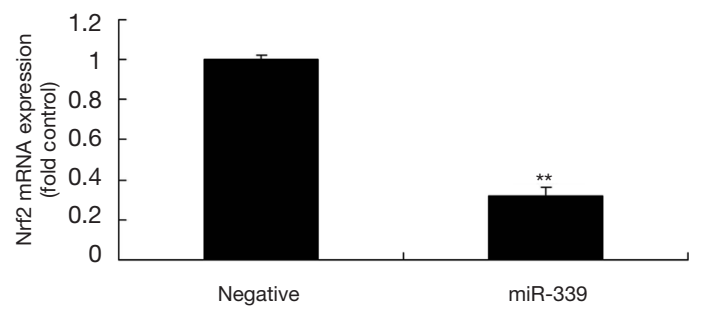

$\mathrm{E}$

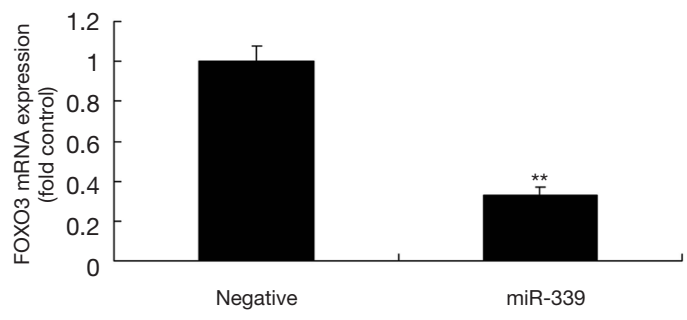

G

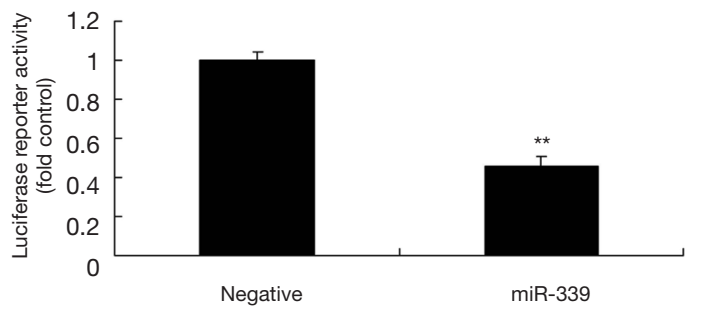

Sirt2 Merge

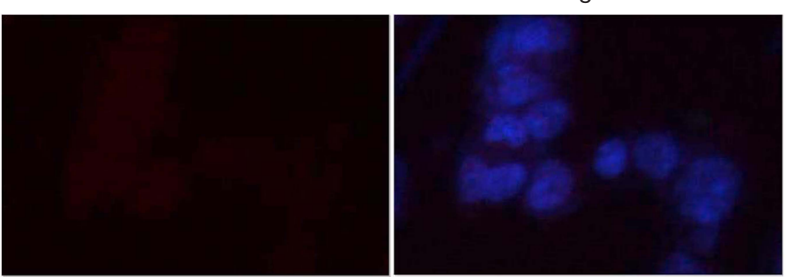

Negative
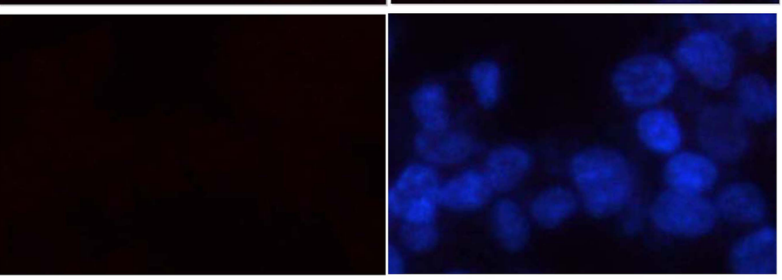

miR-339

Figure 4 Over-expression of miR-339 regulates Sirt2/Nrf2 /FOXO3 in vitro model of CHD. Gene chip and QPCR for Sirt2, Nrf2, HO1, FOXO3 expressions (A-E), Luciferase reporter assay showed that miR-339 targets Sirt2 mRNA (F) and luciferase reporter activity levels (G), IF for Sirt2 expression (H). Negative, negative mimics group; miR-339, over-expression of miR-339 group. **, P<0.01 compared with negative mimics group. Sirt2, silent information regulator 2 protein; Nrf2, nuclear factor E2-related factor 2; HO, heme oxygenase; FOXO3, forkhead box protein O3. 
A

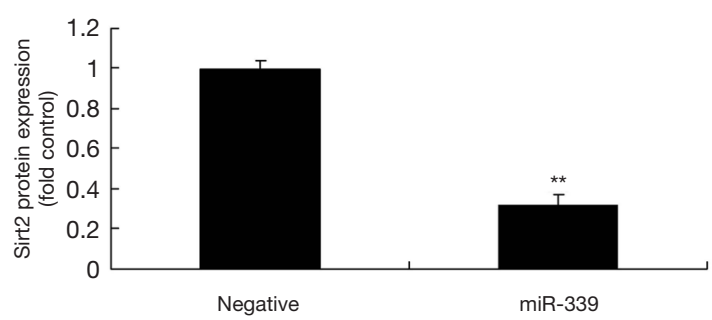

C

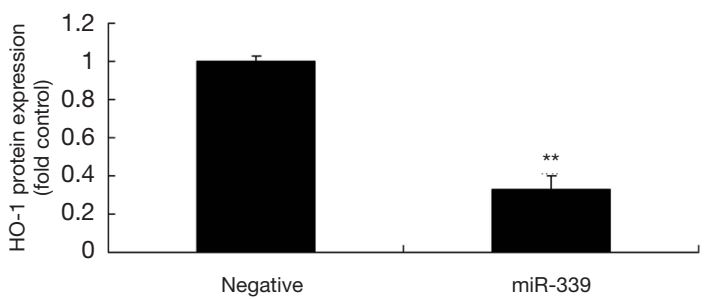

D

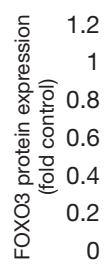

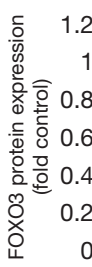

$\mathrm{F}$

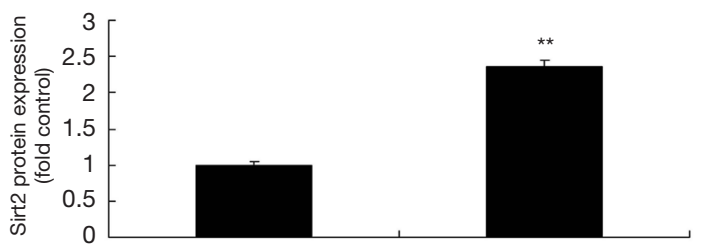

Negative

$\mathrm{H}$
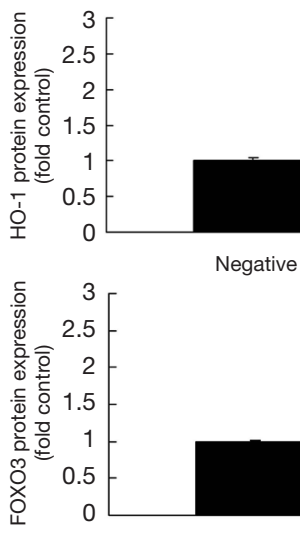

B

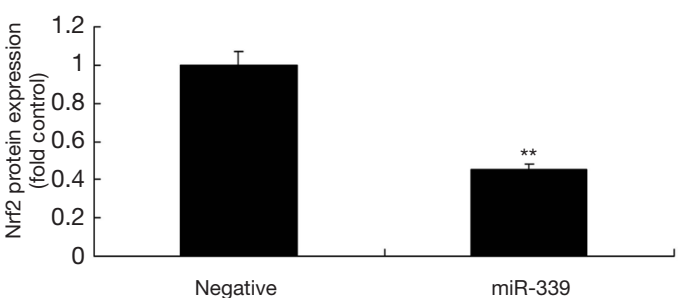

$E$

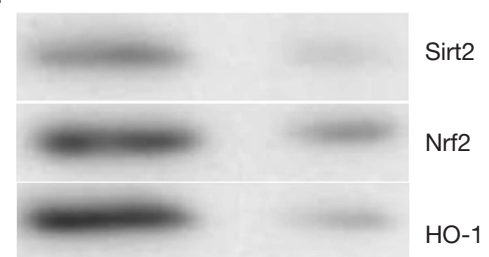

HO-1

FOXO3

GAPDH

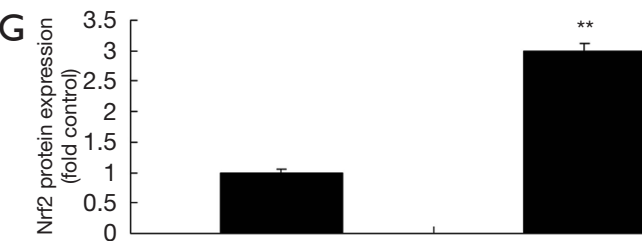

Negative

anti-339

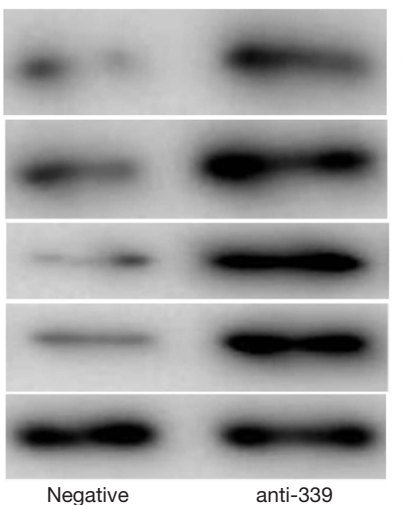

Sirt2

Nrf2

HO-1

FOXO3

GAPDH

Negative

anti-339

Figure 5 MiR-339 regulates Sirt2/Nrf2 /FOXO3 in vitro model of CHD. Sirt2, Nrf2, HO-1, FOXO3 protein expressions by statistical analysis (A,B,C,D) and western blot analysis (E) by over-expression of miR-339; Sirt2, Nrf2, HO-1, FOXO3 protein expressions by statistical analysis (F,G,H,I) and western blot analysis (J) by down-regulation of miR-339. Negative, negative mimics group; miR-339, overexpression of miR-339 group; anti-339, down-regulation of miR-339 group. ** $\mathrm{P}<0.01$ compared with negative mimics group. Sirt2, silent information regulator 2 protein; Nrf2, nuclear factor E2-related factor 2; HO, heme oxygenase; FOXO3, forkhead box protein O3. 
A

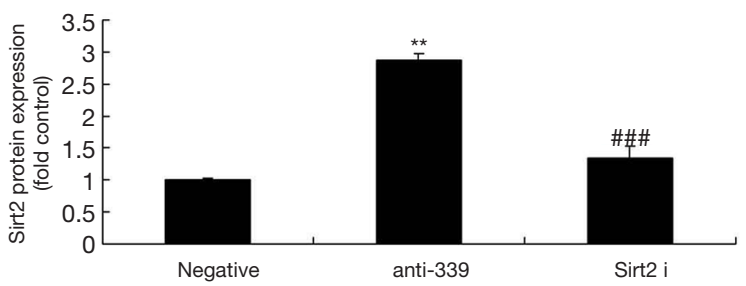

C

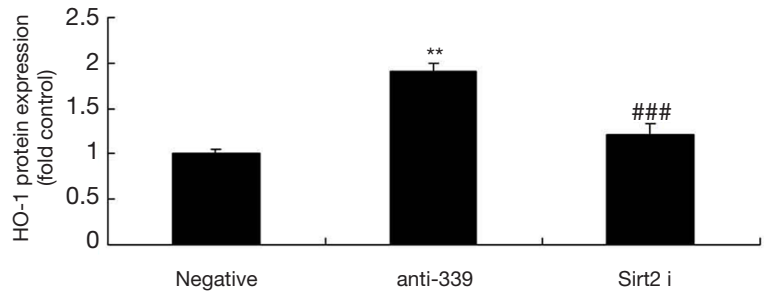

D

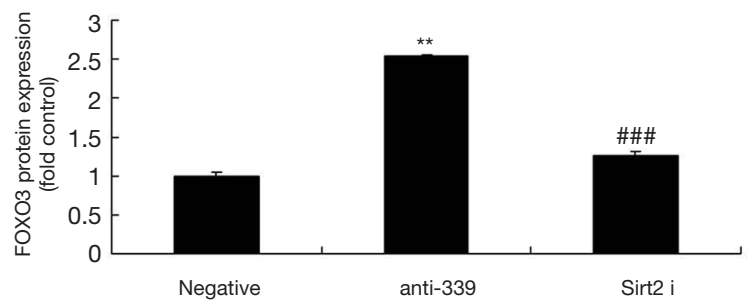

F

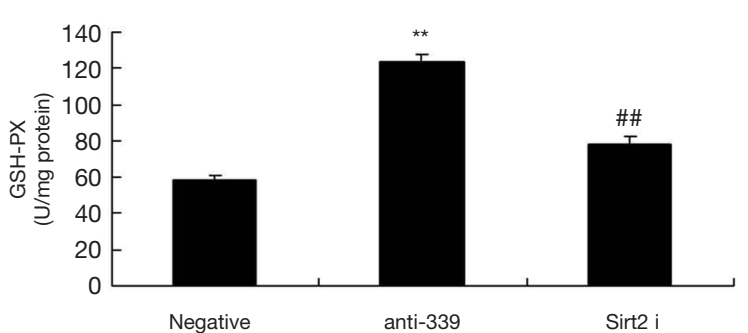

$\mathrm{H}$

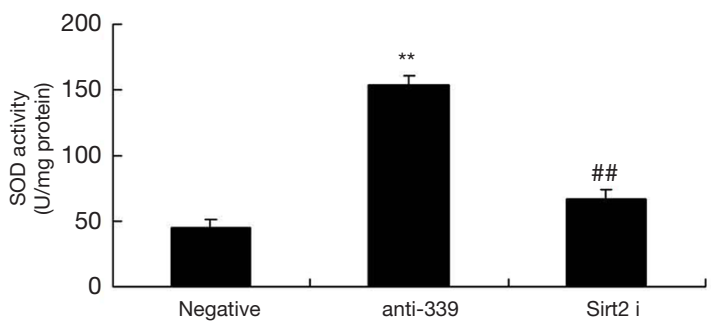

B

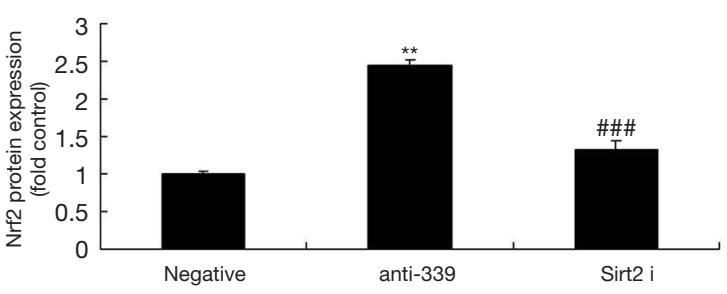

$E$

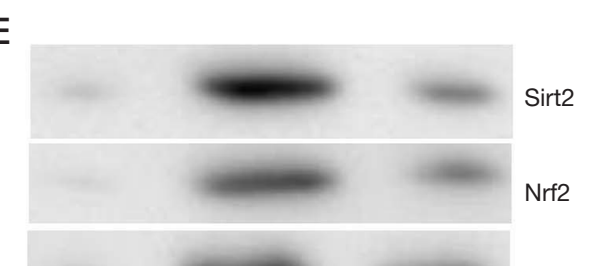

$\mathrm{HO}-1$

FOXO3

GAPDH
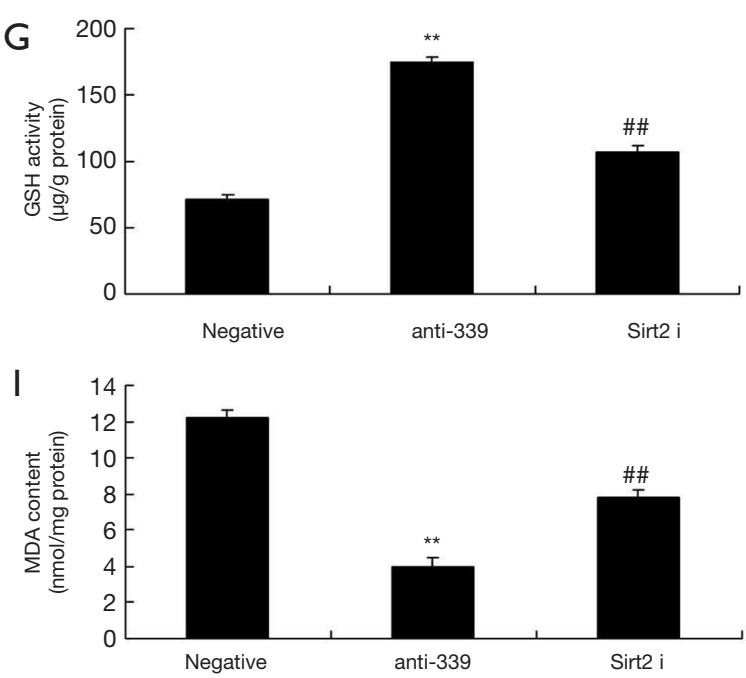

Figure 6 The Sirt2 inhibitor reduced the protective effect of miR-339 down-regulation on oxidative stress in vitro model. Sirt2, Nrf2, HO1, FOXO3 protein expressions by statistical analysis (A,B,C,D) and western blot analysis (E) by over-expression of miR-339; GSH (F), GSH-PX (G), SOD (H), MDA (I). Negative, negative mimics group; anti-339, down-regulation of miR-339 group; Sirt2 i, down-regulation of miR-339 and Sirt2 inhibitor group. ${ }^{* *}, \mathrm{P}<0.01$ compared with negative mimics group; ${ }^{\# \#, ~} \mathrm{P}<0.01$ compared with anti-339 group; GSH, glutathione; SOD, superoxide dismutase; MDA, malondialdehyde; ROS, reactive oxygen species; Sirt2, silent information regulator 2 protein; Nrf2, nuclear factor E2-related factor 2; HO, heme oxygenase; FOXO3, forkhead box protein O3.

The Nvf2 inbibitor reduced the protective effect of miR339 down-regulation on the oxidative stress in vitro model

Then, we used Nrf2 inhibitor to reduce the protein expression of $\mathrm{Nrf2}$ and HO-1 in vitro model of CHD by down-regulation of miR-339, compared with miR-339 down-regulation group (Figure $7 A, B, C$ ). Meanwhile, the inhibition of Nrf2 reduced GSH and GSH-PX levels, and increased MDA levels in vitro model of CHD by downregulation of miR-339, compared with miR-339 down- 

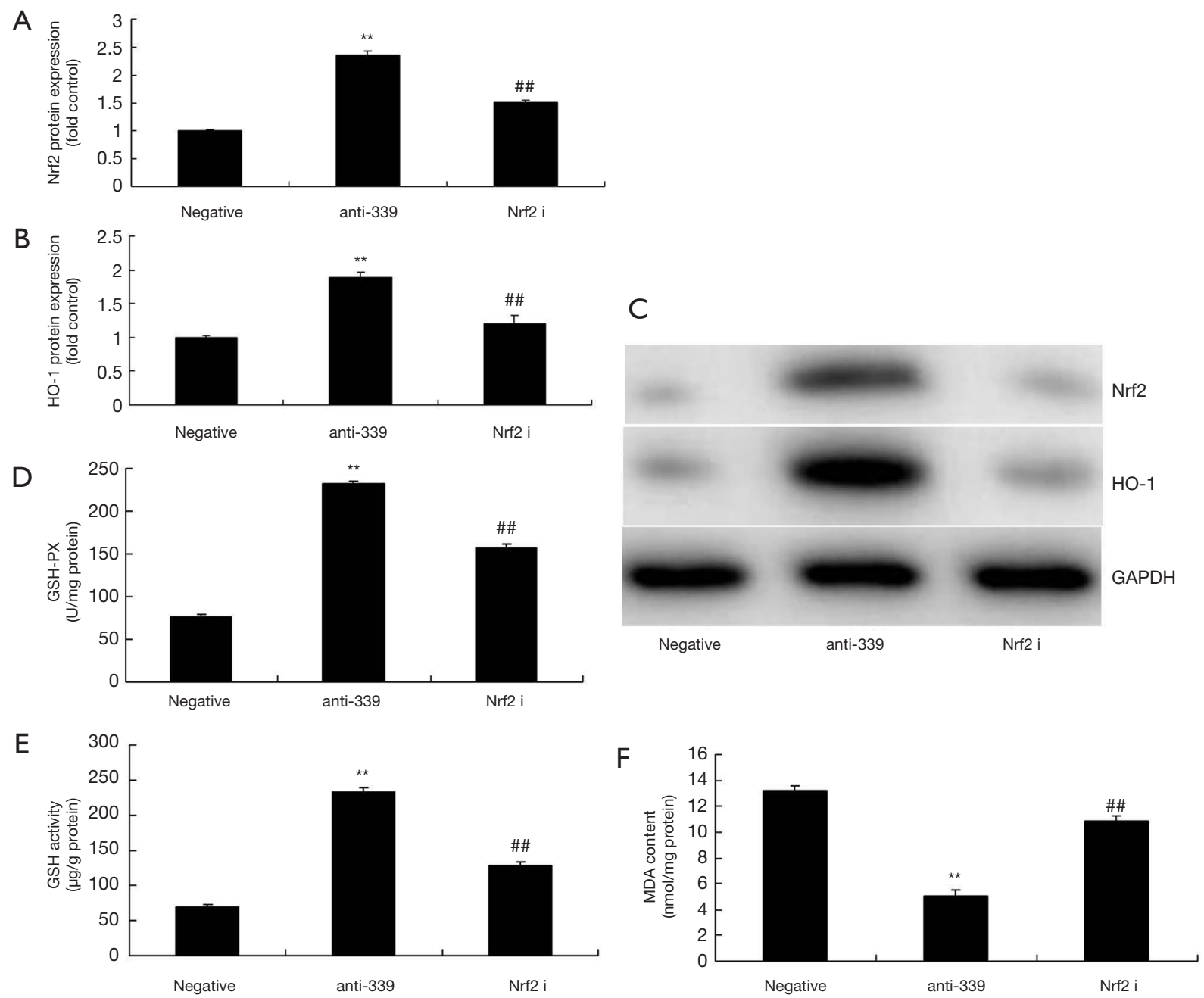

Figure 7 The Nrf2 inhibitor reduced the protective effect of miR-339 down-regulation on oxidative stress in vitro model. Nrf2 and HO-1 protein expressions by statistical analysis (A,B) and western blot analysis (C), GSH (D), GSH-PX (E), MDA (F). Negative, negative mimics group; anti-339, down-regulation of miR-339 group; Nrf2 i, down-regulation of miR-339 and Nrf2 inhibitor group. **, P<0.01 compared with negative mimics group; ${ }^{\# \#}, \mathrm{P}<0.01$ compared with anti-339 group. GSH, glutathione; MDA, malondialdehyde; Nrf2, nuclear factor E2related factor 2; $\mathrm{HO}$, heme oxygenase.

regulation group (Figure 7D,E,F).

compared with miR-339 down-regulation group.

The FOXO3 inbibitor reduced the protective effect of miR339 down-regulation on the oxidative stress in vitro model

Lastly, we used FOXO3 inhibitor to analyze the role of FOXO3 in the protective effect of miR-339 downregulation on oxidative stress in vitro model. As showed in Figure 8, FOXO3 inhibitor reduced the protein expression of FOXO3, reduced SOD levels, and increased MDA levels in vitro model of CHD by miR-339 down-regulation,

\section{Discussion}

CHD is a common disease among the elderly population in China, which is affected by the rapid social economical development and accelerating aging process (3). The morbidity of CHD shows an increasing trend $(12,13)$. At present, CHD has become a frequently occurring disease threatening social public psychological and physiological health. Patients may develop symptoms like chest pain, 


\section{A}
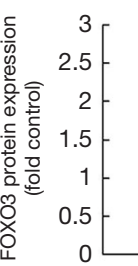

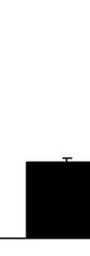

C

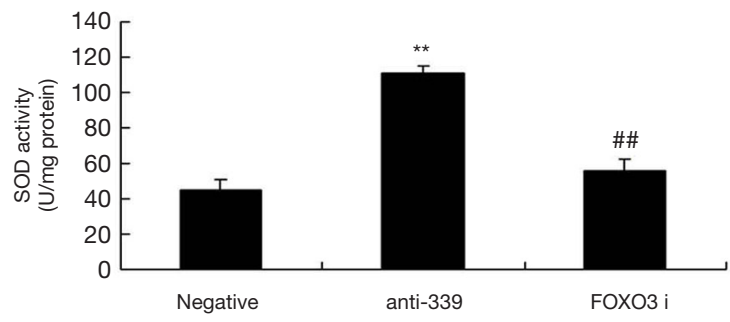

B
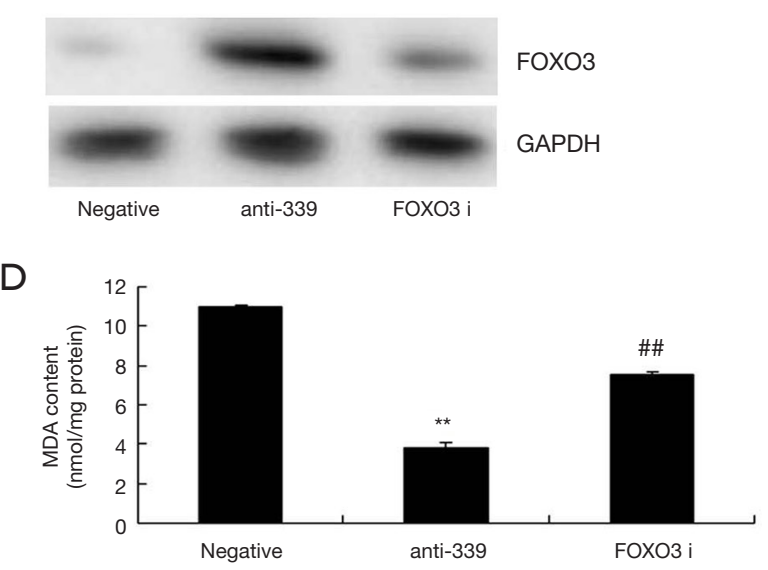

Figure 8 The FOXO3 inhibitor reduced the protective effect of miR-339 down-regulation on oxidative stress in vitro model. FOXO3 protein expressions by statistical analysis (A) and western blot analysis (B), SOD (C), MDA (D). Negative, negative mimics group; anti339, down-regulation of miR-339 group; FOXO3 i, down-regulation of miR-339 and FOXO3 inhibitor group. **, $\mathrm{P}<0.01$ compared with negative mimics group; ${ }^{\#}, \mathrm{P}<0.01$ compared with anti-339 group. SOD, superoxide dismutase; MDA, malondialdehyde; FOXO3, forkhead box protein $\mathrm{O} 3$.

palpitation, and weakness (13). In some severe cases, patient will suffer from acute myocardial infarction (12). Therefore, it has caused great damage to the life health and daily living of patients. On this account, active prevention of CHD to reduce the disease-related injury has become a focus of current medical research (13). In this paper, we firstly found that the serum of miR-339 expression was increased in patients or rats with CHD. Saddic et al. showed that acute human left ventricular ischemia upregulated miRNAs miR339-5p and miR-483-3p and the downregulated miRNA miR-139-5p (14).

Endothelial structural and functional impairments are also involved in the formation and qualitative changes of atheromatous plaque (15). The excessive activation of the oxidative stress response is an important step leading to endothelial injury (5). ROS is an important catalyzing enzyme in the body, which can catalyze the production of oxygen free radicals and mediate the activation of oxidative stress response (16). High ROS expression can increase the production of oxygen free radicals and act on the endothelial cell (17). On the one hand, it can result in increased production of lipid oxidation product MDA. On the other hand, it can result in elevated production of endothelial injury marker ET-1 (15). Sirt2 and SOD are the antioxidants exerting protective effect during endothelial oxidative injury (18). The former can reduce the production of oxygen free radical through the Nrf2/
FOXO3 pathway, while the latter can scavenge the oxygen free radical through reduction reaction (19). Our findings implied that over-expression of miR-339 increased oxidative stress and not affected on ROS levels in vitro model.

SIRT2 participates in multiple pathophysiological processes, including aging, energy restriction, oxidative stress, cell apoptosis and inflammatory response (7). Besides, it also plays a role in mitochondriogenesis and biological circadian rhythm regulation. The latest study finds that SIRT2 is related to the genesis of multiple tumors, nervous system-related degenerative disease, aging-related disease and cardiovascular disease (20). It exerts its function through signal pathways such as FOXO transcription factor, Nrf2, PEPCK1 and p65 (19). Our present data showed that over-expression of miR-339 suppressed Sirt2, Nrf2, HO-1 and FOXO3 protein expressions in vitro model. Meanwhile, the Sirt2 inhibitor reduced the protective effect of miR-339 down-regulation on oxidative stress in vitro model. Wang et al. suggested that miR-339/Sirt2/NF-кB/FOXO1 was keeping in the effects of acupuncture in SH-SY5Y cells and rat PC12 cells (21).

Recently, reports have reported the biological function of SIRT2 in oxidative stress. Oxidative stress will up-regulate SIRT2 expression in NIH3T3 cells due to the elevated hydrogen peroxide level (20). In addition, it can activate the $\mathrm{FOXO} 3$ gene through deacetylation, which can thereby 


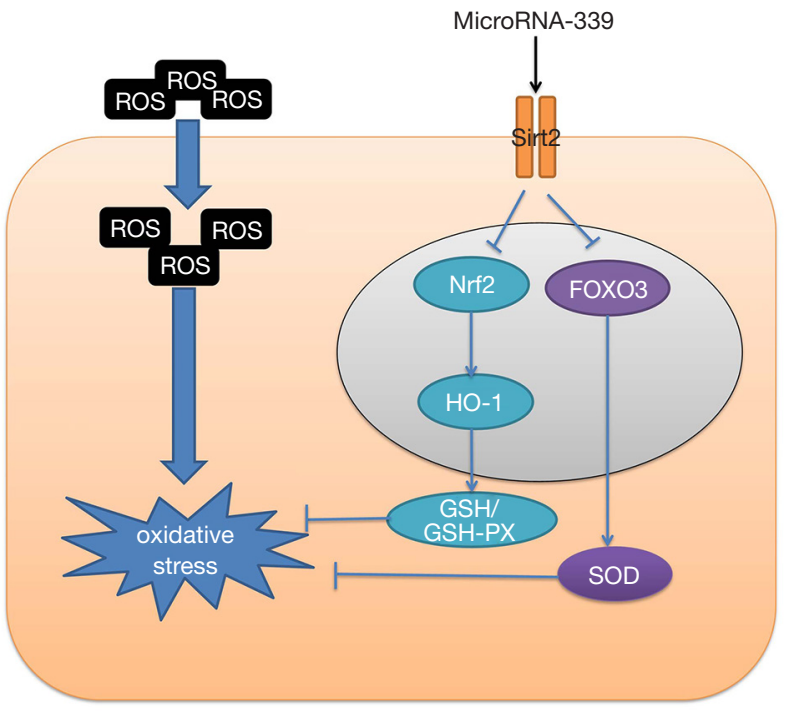

Figure 9 MiR-339 is a Potential Biomarker of CHD to aggravate oxidative stress through $\mathrm{Nrf2/FOXO3} \mathrm{targeting} \mathrm{Sirt2.} \mathrm{CHD,}$ coronary heart disease; Nrf2, nuclear factor E2-related factor 2; Sirt2, silent information regulator 2 protein; FOXO3, forkhead box protein $\mathrm{O} 3$.

up-regulate MnSOD and reduce the ROS level (19). In mouse macrophage-like cell lines, the pep-1-sirt2 protein with cell permeation function can induce lipopolysaccharide and $\mathrm{H}_{2} \mathrm{O}_{2}$ related death in mouse cells (22). FOXO3 is an important protein family system for regulating cell apoptosis, cell cycle, DNA damage repair, oxidative stress, energy metabolism, longevity, cancer genesis and development, and other cellular functions (22). Our data also showed that FOXO3 inhibitor reduced the protective effect of miR-339 down-regulation on oxidative stress in vitro model.

$\mathrm{Nrf} 2$ is a critical transcription factor for regulating the expression of multiple antioxidase genes. For instance, HO-1 is an enzyme with antioxidation and cell protection. The activation of the Nrf2/HO-1 signal can protect the cell from oxidative injury (23). HO-1 is an important antioxidase that mainly catalyzes the decomposition and metabolism of heme into ferrous, carbon monoxide and biliverdin (23). On the one hand, heme group degradation contributes to preventing its oxidation promoting effect (24). On the other hand, the by-product biliverdin, together with the reduced form bilirubin, has effective ROS scavenging activity (24). They can defend against peroxide, peroxynitrite, hydroxyl, and superoxide free radical (24). Thus, our data demonstrated that the Nrf2 inhibitor reduced the protective effect of miR-339 down-regulation on oxidative stress in vitro model (Figure 9).

Therefore, our study provided novel insight into the pathological mechanisms of CHD, and miR-339 is a potential biomarker of CHD to aggravate oxidative stress through Nrf2/FOXO3 targeting Sirt2. Although our study confirmed miR-339 is a potential biomarker of CHD, the target genes of miR-339 or other differentially expressed are also necessary to be carefully tested for CHD.

\section{Conclusions}

Our study has revealed that Over-expression of miR-339 increased oxidative stress and not affected on ROS levels. MiR-339 is a Potential Biomarker of CHD to aggravate oxidative stress through Nrf2/FOXO3 targeting Sirt2. MiR-339/Sirt2 may be considered as the new targets for the treatment of CHD.

\section{Acknowledgments}

Funding: None.

\section{Footnote}

Reporting Checklist: The authors have completed the ARRIVE reporting checklist. Available at http://dx.doi. org/10.21037/apm-20-603

Data Sharing Statement: Available at http://dx.doi. org/10.21037/apm-20-603

Conflicts of Interest: All authors have completed the ICMJE uniform disclosure form (available at http://dx.doi. org/10.21037/apm-20-603). The authors have no conflicts of interest to declare.

Ethical Statement: The authors are accountable for all aspects of the work in ensuring that questions related to the accuracy or integrity of any part of the work are appropriately investigated and resolved. The study was conducted in accordance with the Declaration of Helsinki (as revised in 2013). The study was approved by ethics board of Beijing Chaoyang Hospital, Capital Medical University (No.: 2016-6-4-1). Experiments were performed under a project license granted by ethics board of Beijing Chaoyang Hospital, Capital Medical University (approval NO: F2016025), in compliance with institutional guidelines for the care 
and use of animals.

Open Access Statement: This is an Open Access article distributed in accordance with the Creative Commons Attribution-NonCommercial-NoDerivs 4.0 International License (CC BY-NC-ND 4.0), which permits the noncommercial replication and distribution of the article with the strict proviso that no changes or edits are made and the original work is properly cited (including links to both the formal publication through the relevant DOI and the license). See: https://creativecommons.org/licenses/by-nc-nd/4.0/.

\section{References}

1. Brandao SMG, Hueb W, Ju YT, et al. Utility and qualityadjusted life-years in coronary artery disease: Five-year follow-up of the MASS II trial. Medicine (Baltimore) 2017;96:e9113.

2. Hirji SA, Stevens SR, Shaw LK, et al. Predicting risk of cardiac events among ST-segment elevation myocardial infarction patients with conservatively managed noninfarct-related artery coronary artery disease: An analysis of the Duke Databank for Cardiovascular Disease. Am Heart J 2017;194:116-24.

3. Raungaard B, Christiansen EH, Botker HE, et al. Comparison of Durable-Polymer Zotarolimus-Eluting and Biodegradable-Polymer Biolimus-Eluting Coronary Stents in Patients With Coronary Artery Disease: 3-Year Clinical Outcomes in the Randomized SORT OUT VI Trial. JACC Cardiovasc Interv 2017;10:255-64.

4. Mahalakshmi A, Kurian GA. Evaluating the impact of diabetes and diabetic cardiomyopathy rat heart on the outcome of ischemia-reperfusion associated oxidative stress. Free Radic Biol Med 2018;118:35-43.

5. Shahsavari G, Raoufi A, Toolabi A, et al. The effect of atorvastatin treatment duration on oxidative stress markers and lipid profile in patients with coronary artery diseases: A case series study. ARYA Atheroscler 2017;13:282-7.

6. Chen CJ, Yu W, Fu YC, et al. Resveratrol protects cardiomyocytes from hypoxia-induced apoptosis through the SIRT1-FoxO1 pathway. Biochem Biophys Res Commun 2009;378:389-93.

7. Yang $\mathrm{W}$, Gao F, Zhang P, et al. Functional genetic variants within the SIRT2 gene promoter in acute myocardial infarction. PLoS One 2017;12:e0176245.

8. Tong KL, Mahmood Zuhdi AS, Wan Ahmad WA, et al. Circulating MicroRNAs in Young Patients with Acute Coronary Syndrome. Int J Mol Sci 2018;19:1467.
9. Marí-Alexandre J, Barceló-Molina M, Sanz-Sánchez J, et al. Thickness and an Altered miRNA Expression in the Epicardial Adipose Tissue Is Associated With Coronary Heart Disease in Sudden Death Victims. Rev Esp Cardiol (Engl Ed) 2019;72:30-9.

10. Bildirici AE, Arslan S, Özbilüm Şahin N, et al. MicroRNA-221/222 expression in atherosclerotic coronary artery plaque versus internal mammarian artery and in peripheral blood samples. Biomarkers 2018;23:670-5.

11. $\mathrm{Xu} \mathrm{X,} \mathrm{Li} \mathrm{H.} \mathrm{Integrated} \mathrm{microRNA-gene} \mathrm{analysis} \mathrm{of}$ coronary artery disease based on miRNA and gene expression profiles. Mol Med Rep 2016;13:3063-73.

12. Möbius-Winkler S, Uhlemann M, Adams V, et al. Coronary Collateral Growth Induced by Physical Exercise: Results of the Impact of Intensive Exercise Training on Coronary Collateral Circulation in Patients With Stable Coronary Artery Disease (EXCITE) Trial. Circulation 2016;133:1438-48.

13. Dillinger JG, Maher V, Vitale C, et al. Impact of Ivabradine on Central Aortic Blood Pressure and Myocardial Perfusion in Patients With Stable Coronary Artery Disease. Hypertension 2015;66:1138-44.

14. Saddic LA, Chang TW, Sigurdsson MI, et al. Integrated microRNA and mRNA responses to acute human left ventricular ischemia. Physiol Genomics 2015;47:455-62.

15. Kuliczkowski W, Golanski R, Bijak M, et al. Relationship between high on aspirin platelet reactivity and oxidative stress in coronary artery by-pass grafted patients. Blood Coagul Fibrinolysis 2016;27:151-5.

16. Mazereeuw G, Herrmann N, Andreazza AC, et al. Baseline Oxidative Stress Is Associated with Memory Changes in Omega-3 Fatty Acid Treated Coronary Artery Disease Patients. Cardiovasc Psychiatry Neurol 2017;2017:3674371.

17. Shahid SU, Shabana, Humphries S. The SNP rs10911021 is associated with oxidative stress in coronary heart disease patients from Pakistan. Lipids Health Dis 2018;17:6.

18. Sarikhani M, Mishra S, Desingu PA, et al. SIRT2 regulates oxidative stress-induced cell death through deacetylation of c-Jun NH2-terminal kinase. Cell Death Differ 2018;25:1638-56.

19. Wang F, Chan CH, Chen K, et al. Deacetylation of FOXO3 by SIRT1 or SIRT2 leads to Skp2-mediated FOXO3 ubiquitination and degradation. Oncogene 2012;31:1546-57.

20. Tang X, Chen XF, Wang NY, et al. SIRT2 Acts as a Cardioprotective Deacetylase in Pathological Cardiac Hypertrophy. Circulation 2017;136:2051-67. 
21. Wang JY, Li H, Ma CM, et al. Acupuncture may exert its therapeutic effect through microRNA-339/Sirt2/NFkB/ FOXO1 axis. Biomed Res Int 2015;2015:249013.

22. Willcox BJ, Morris BJ, Tranah GJ, et al. LongevityAssociated FOXO3 Genotype and its Impact on Coronary Artery Disease Mortality in Japanese, Whites, and Blacks: A Prospective Study of Three American Populations. J Gerontol A Biol Sci Med Sci 2017;72:724-8.

Cite this article as: Shi L, Zhang Y, Zhang J, Gao Y, Liu J, Chen M, Yang X. MiR-339 is a potential biomarker of coronary heart disease to aggravate oxidative stress through Nrf2/ FOXO3 targeting Sirt2. Ann Palliat Med 2021;10(3):25962609. doi: 10.21037/apm-20-603
23. Donovan EL, McCord JM, Reuland DJ, et al. Phytochemical activation of Nrf2 protects human coronary artery endothelial cells against an oxidative challenge. Oxid Med Cell Longev 2012;2012:132931.

24. Yu H, Shi L, Zhao S, et al. Triptolide Attenuates Myocardial Ischemia/Reperfusion Injuries in Rats by Inducing the Activation of Nrf2/HO-1 Defense Pathway. Cardiovasc Toxicol 2016;16:325-35. 